\title{
ON SOME FORMULAS ABOUT VOLUME AND SURFACE AREA
}

\author{
MINORU KURITA
}

We prove in this paper some integral formulas about volume and surface area which are the extensions of the classical formulas such as Guldin-Pappus's theorem about the solid of rotation and the surface of rotation and Holditch's theorem about the area of the domains bounded by the loci of three points on a segment that moves on the euclidean plane. The formulas we prove are so elementary that they may be found in some literature, but the proofs here given are very simple by the use of moving frames and I assume that they are of some interest.

\section{The generalization of Guldin-Pappus's theorem}

1.1 Let the volume of a solid $M$ in the $n$-dimensional euclidean space be $V$. We cut the solid $M$ by one-parametric continuously difierentiable set of hyperplanes such that through each point of $M$ one and only nne of the hyperplanes passes and let $v$ be an $n-1$ dimensional volume of the section. We assume moreover that the locus of the center of gravity of the section of $M$ by each one of the hyperplanes is a curve with continuous tangents with respect to the parameter $t$ of the hyperplanes, and let $d \sigma$ be an orthogonal component of an arc element of the locus of the center of gravity of the section to the normal direction of the section. Then we have

$$
V=\int v d \sigma .
$$

Proof. We can take a rectangular frame $R^{\prime}$ with an origin $\mathbf{A}^{\prime}$ at the center of gravity of the section and $n$-th fundamental vector $\mathbf{e}_{n}^{\prime}$ on the normal of the section on the side for which the parameter $t$ of the locus of the center of gravity increases, in such a way that the set of these frames is continuously differentiable with respect to $t$. Let the origin of the fundamental rectangular frame $R_{0}$ be $\mathbf{A}^{0}$ and $n$ fundamental vectors be $\mathbf{e}_{1}^{0}, \ldots, \mathbf{e}_{n}^{0}$.

We put $\mathbf{A}^{\prime}=\mathbf{A}^{0}+\sum_{i=1}^{n} p_{i} \mathbf{e}_{i}^{0}, \mathbf{e}_{i}^{\prime}=\sum_{j=1}^{n} p_{i} \mathbf{e}_{j}^{0}$. Let the frame got by translation from $R^{\prime}$ along the section be $R$ and its origin and vectors be $\mathbf{A}, \mathbf{e}_{1}, \mathbf{e}_{2}, \ldots$, $\mathbf{e}_{n}$. Then we have $\mathbf{A}=\mathbf{A}^{\prime}+\sum_{i=1}^{n-1} x_{i} \mathbf{e}_{i}^{\prime}, \mathbf{e}_{i}=\mathbf{e}_{i}^{\prime}$. Now we put

Received May 14, 1953. 


$$
R_{0}=\left(\begin{array}{c}
\mathbf{A}^{0} \\
\mathbf{e}_{1}^{0} \\
\vdots \\
\vdots \\
\mathbf{e}_{n}^{0}
\end{array}\right), \quad R^{\prime}=\left(\begin{array}{c}
\mathbf{A}^{\prime} \\
\mathbf{e}_{1}^{\prime} \\
\vdots \\
\vdots \\
\mathbf{e}_{n}^{\prime}
\end{array}\right), \quad R=\left(\begin{array}{c}
\mathbf{A} \\
\mathbf{e}_{1} \\
\vdots \\
\vdots \\
\mathbf{e}_{n}
\end{array}\right)
$$

and let the matrices of transformations between them be $P, T$. Then we have $R^{\prime}=P R_{0}, R=T R^{\prime}$ and hence

$$
R=T P R_{0}
$$

where

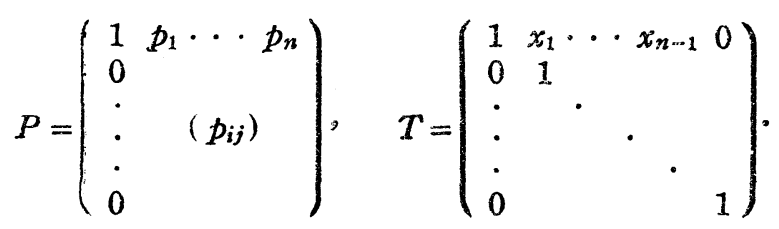

The coefficients $\omega_{i}, \omega_{i j}$ of the infinitesimal relative displacement of the motion of $R$ are obtained as the coefficients of the matrix

$$
d(T P)(T P)^{-1}=d T \cdot T^{-1}+T\left(d P P^{-1}\right) T^{-1} .
$$

Let the infinitesimal relative displacement of $R^{\prime}$ be given by $d \mathrm{~A}^{\prime}=\sum_{i=1}^{n} \pi_{i} \mathrm{e}_{i}^{\prime}, d \mathbf{e}_{i}^{\prime}$ $=\sum_{j=1}^{n} \pi_{i j} \mathrm{e}_{j}^{\prime}$. Then $\pi_{i}, \pi_{i j}$ are the coefficierits of $d P P^{-1}$. Calculating (3) we get

$$
\begin{aligned}
& \omega_{i}=d x_{i}+\pi_{i}+\sum_{j=1}^{n-1} x_{j \pi j i} \quad(i=1, \ldots, n-1) \\
& \omega_{n}=\pi_{n}+\sum_{j=1}^{n-1} x_{j \pi j n} .
\end{aligned}
$$

We denote by $d V$ a volume element of the solid $M$. As $\pi i, \pi_{i j}$ are linear differential forms with a single variable $t$ we get

$$
d V=\left[\omega_{1} \ldots \omega_{n}\right]=\left[d x_{1} \ldots d x_{n-1}, \pi n+\sum_{i=1}^{n-1} x_{i} \pi_{i n}\right] .
$$

Since $\mathbf{A}$ is a center of the gravity of the section we have

$$
\int x_{i} d x_{1} \ldots d x_{n-1}=0 \quad(i=1, \ldots, n-1)
$$

Hence we get (1) when we remark $\pi_{n}=d \sigma$.

As a special case we consider a tube. We mean by 'tube' a solid bounded by orthogonal trajectories of one-parametric continuously differentiable set of hyperplanes and two of the hyperplanes with the assumption that any two of 
the hyperplanes do not intersect each other in the solid. It is well known that all orthogonal sections are congruent (Cf. [2] p. 117). Our definition of a tube is different from that of the paper [1]. The volume of a tube is given by

$$
V=v l \text {, }
$$

where $v$ is an $n-1$ dimensional volume of the orthogonal section and $l$ is an arc-length of the locus of the center of gravity of the section. This is a generalization of Guldin-Pappus's theorem.

1.2. As to surface area we can not obtain a formula analogous to (1), but the one analogous to (5) can be obtained. We cut an $n-1$ dimensional surface $M$ by one-parametric set of hyperplanes which have the property stated in $\mathbf{1 . 1}$ and let a surface element of $M$ be $d S$. Then we have

$$
d S^{2}=\left[\omega_{1} \ldots \omega_{n-1}\right]^{2}+\left[\omega_{1} \ldots \omega_{n-2} \omega_{n}\right]^{2}+\ldots+\left[\omega_{2} \ldots \omega_{n}\right]^{2} .
$$

In our case variables $x_{1}, \ldots, x_{n-1}, t$ are not independent contrary to the case stated in 1.1. $x_{1}, \ldots, x_{n-1}$ are functions of $t$ and $n-2$ variables $u_{1}, \ldots, u_{n-2}$ which determine the position of a point on the hyperplane of the section. If we denote by attaching 0 to a differential form the one got by putting $t$ into a constant, then we have

$$
\left[d x_{1} \ldots d x_{n-2}\right]_{0}^{2}+\ldots+\left[d x_{2} \ldots d x_{n-1}\right]_{0}^{2}=C(u)^{2}\left[d u_{1} \ldots d u_{n-2}\right]^{2} .
$$

As $C(u)\left[d u_{1} \ldots d u_{n-2}\right]$ is a surface element $d s$ of a section of $M$ by a hyperplane, we can put

$$
\left[d x_{2}, \ldots d x_{n-1}\right]_{0}=c_{1} d s, \ldots,\left[d x_{1} \ldots d x_{n-2}\right]_{0}=c_{n-1} d s
$$

where $\sum_{i=1}^{n-1} c_{i}^{?}=1$. Hence the right side of (6) with the exception of the first term is equal to

$$
\begin{aligned}
& {\left[\left[d x_{1} \ldots d x_{n-2}\right]_{0} \omega_{n}\right]^{2}+\ldots+\left[\left[d x_{2} \ldots d x_{n-1}\right]_{0} \omega_{n}\right]^{2} } \\
= & {\left[c_{n-1} d s, \omega_{n}\right]^{2}+\ldots+\left[c_{1} d s, \omega_{n}\right]^{2}=\left[d s, \omega_{n}\right]^{2} . }
\end{aligned}
$$

Hence (6) can be written as

$$
d S^{2}=\left[\omega_{1} \ldots \omega_{n-1}\right]^{2}+\left[d s, \pi_{n}+\sum_{j=1}^{n-1} x_{j \pi j n}\right]^{2} .
$$

Now we assume $M$ is an $n-1$ dimensional surface generated by orthogonal trajectories of one-parametric continuously differentiable set of hyperplanes and is cut by two of the hyperplanes. Then all the sections are congruent and if we take a suitable frame with origin at the center of gravity of the section, 
$x_{1}, \ldots, x_{n-1}$ are functions of $n-2$ variables which are independent with $t$ and moreover we have

$$
\pi_{i}=0, \quad \pi_{i j}=0 \quad(i, j=1, \ldots, n-1) .
$$

Hence we get by virtue of (4) $\omega_{1}=d x_{1}, \ldots, \omega_{n-1}=d x_{n-1}$, hence $\left[\omega_{1} \ldots \omega_{n-1}\right]$ $=0$. As the origin is at the center of gravity we have $\int x_{i} d s=0 \quad(i=1, \ldots$, $n-1)$. Thus we obtain $d S=\left[d s, \omega_{n}\right]$. Integrating this we get

$$
S=s l \text {, }
$$

where $S$ is a surface area of $M, s$ is an $n-2$ dimensional area of the section and $l$ is an arc-length of the locus of the center of gravity of the section. This is a generalization of Guldin-Pappus's theorem.

1.3 Next we treat the case of spherical space. Let the volume of a solid $M$ in the $n$-dimensional spherical space with the radius 1 be $V$. We cut $M$ by one-parametric continuously differentiable set of $n-1$ dimensional spheres of radius 1 which have the property stated at the beginning of 1.1. Let $R_{0}$ be a fundamental rectangular frame and $R^{\prime}$ be a frame with the ends of first $n$ fundamental vectors $\mathbf{e}_{n}^{\prime}, \mathbf{e}_{1}^{\prime}, \ldots, \mathbf{e}_{n-1}^{\prime}$ on the $n-1$ dimensional sphere of the section. We assume $R^{\prime}$ is defined uniquely for each section and let $R$ be any frame with the ends of $\mathbf{e}_{0}, \mathbf{e}_{1}, \ldots, \mathbf{e}_{n-1}$ on the section. Then putting

$$
R_{3}=\left(\begin{array}{c}
\mathbf{e}_{0}^{0} \\
\mathbf{e}_{1}^{0} \\
\vdots \\
\vdots \\
\mathbf{e}_{n}^{0}
\end{array}\right), \quad R^{\prime}=\left(\begin{array}{c}
\mathbf{e}_{0}^{\prime} \\
\mathbf{e}_{1}^{\prime} \\
\vdots \\
\vdots \\
\mathbf{e}_{n}^{\prime}
\end{array}\right), \quad R=\left(\begin{array}{c}
\mathbf{e}_{0} \\
\mathbf{e}_{1} \\
\cdot \\
\cdot \\
\mathbf{e}_{n}
\end{array}\right)
$$

we get $R^{\prime}=P R_{0}, R=T R^{\prime}$ and hence $R=T P R_{0}$, where $T$ is a matrix such that

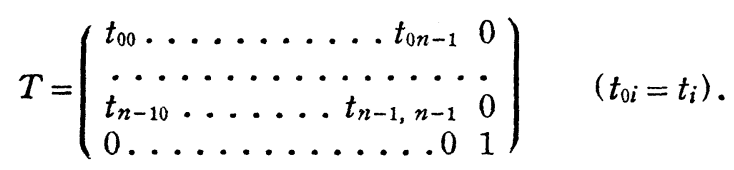

The first row of $d T T^{-1}$ is of the form $\left(0, \tau_{1}, \ldots, \tau_{n-1}, 0\right)$. Let the parameter of the sectional $n-1$ dimensional spheres be $t$. Then the coefficients of $d P P^{-1}$, namely $\pi_{i j}(i, j=0,1, \ldots, n)$ when we put $d \mathrm{e}_{i}^{\prime}=\sum_{j=0}^{n} \pi_{i j} \mathbf{e}_{j}^{\prime}$, are linear differential forms with a single variable $t$. If we put $d \mathbf{e}_{i}=\sum_{j=0}^{n} \omega_{i j} \mathbf{e}_{j}\left(\omega_{0 i}=\omega_{i}\right), \omega_{i j}$ 's are the coefficients of $d(T P)(T P)^{-1}$ and we get as in $\mathbf{1 . 1}$

$$
\begin{aligned}
\omega_{i} & \equiv \tau_{i} \quad(\bmod . d t, i=1, \ldots, n-1) \\
\omega_{n} & =\sum_{i=0}^{n-1} t_{i} \pi_{i n} .
\end{aligned}
$$


Hence the volume element $d V$ of the solid generated by the endpoint of $\mathbf{e}_{0}$ is given by

$$
d V=\left[\omega_{1} \ldots \omega_{n-1} \omega_{n}\right]=\left[\tau_{1} \ldots \tau_{n-1}, \sum_{i=0}^{n-1} t_{i} \pi_{i n}\right] .
$$

Since $d v=\left[\tau_{1} \ldots \tau_{n-1}\right]$ is a volume element of a section we can write

$$
d V=\sum_{i=0}^{n-1}\left[t_{i} d v, \pi_{i n}\right]
$$

Now we consider the integrals $T_{i}=\int t_{i} d v(i=0,1, \ldots, n-1)$ over the section. $\left(T_{0}, T_{1}, \ldots, T_{n-1}\right)$ are components of a vector defired by the section and its length

$$
w=\left(\sum_{i=0}^{n-1} T_{i}^{2}\right)^{1 / 2}
$$

is a geometric quantity attached to the section which is different from the volume. As $\left(T_{0}, T_{1}, \ldots, T_{n-1}\right)$ are components of a vector we can put $T_{1}$ $=\ldots=T_{n-1}=0$ by taking $R^{\prime}$ suitably. We call a center of graviity of the section the endpoint of $\mathbf{e}_{0}^{\prime}$. Then we get $w=\int t_{1} d v, \int t_{i} d v=0 \quad(i=1,2, \ldots$, $n-1)$. Hence we get by virtue of $(8)$

$$
V=\int w d \sigma
$$

where $d \sigma=\pi_{0 n}$ is an orthogonal component of an arc-element of the locus of the center of gravity of the section to the direction of the normal of the section. It is notable that $w$ in (10) is not the volume of the section. A tube can be defined in our space as in $\mathbf{1 . 1}$ (cf. [2] p. 117), and a formula $V=w l$ analogous to (5) can be obtained.

In the special case $n=2$ a solid $M$ is a domain on the sphere in the euclidean space of dimension 3 , and we cut $M$ by great circles which do not cut in the domain $M$ each other. It can easily be verified that the center of gravity of the arc of section is its middle point and $w$ is a length of a chord corresponding to the arc. $d \sigma$ is an orthogonal component of an arc element of the locus of the middle points of the arcs of section to the normal direction of the arc. If one end of each arc is fixed at $O$, we have for a spherical area $S$ a well known formula $S=\int 1 / 2 l^{2} d c$ where $l$ is a length of a chord and $d \varphi$ is an infinitesimal angle between two consecutive planes containing the chords and the diameter of the sphere through $O$.

1.4 We can obtain a formula analogous to $(7)$ in the spherical case. Let $M$ be an $n-1$ dimensional surface in the spherical space of dimension $n$ with the radius 1 generated by orthogonal trajectories of one-parametric set of the 
$n-1$ dimensional sphere of radius 1 and cut by two of the spheres. Let the equation of the $n-1$ dimensional sphere be $\sum_{i=0}^{n-1} t_{i}^{2}=1$ and the surface element of the section be $d S$. We put $U_{i}=\int t_{i} d s(i=0,1, \ldots, n-1)$ and $u=\left(\sum_{i=0}^{n-1} U_{i}^{2}\right)^{1 / 2}$ and call a center of gravity the point with $\left(U_{\mathrm{c}} u^{-1}, U_{1} u^{-1}, \ldots, U_{n-1} u^{-1}\right)$ as its coordinates. Then the surface area $S$ of $M$ is given by

$$
S=u l
$$

where $l$ is a length of the locus of a center of gravity. The proof is analogous to that of $\mathbf{1 . 2}$.

\section{Generalized Holditch's theorem}

2.1 Let a straight line move in the $n$-dimensional euclidean space and let local parameters of this motion be $u_{1}, \ldots, u_{n-1}$. We take on this line a point $\mathbf{A}$ and a unit vector $\mathbf{e}_{1}$ and let $R$ be a rectangular frame with origin at $\mathbf{A}$ and. one of its fundamental vectors $\mathbf{e}_{1}, \ldots, \mathbf{e}_{n}$ on $\mathbf{e}_{1}$. We take on each line a fixed point $\mathbf{A}^{\prime}$ and let $R^{\prime}$ be a frame wirh $\mathbf{A}^{\prime}$ as its origin and $\mathbf{e}_{i}^{\prime}=\mathbf{e}_{i}$ as its vectors. Then $\mathrm{A}=\mathbf{A}^{\prime}+t \mathbf{e}_{1}, \mathbf{e}_{i}=\mathbf{e}_{i}^{\prime}$, and putting $R=T R^{\prime}, R^{\prime}=P R_{0}$, where $R$, is a fundamental frame, we have $R=T P R_{0}$. Hence the coefficients $\omega_{i}, \omega_{i j}$ of the infinitesimal relative displacement of $R$ are given by calculating $d(T P)(T P)^{-1}$. Writing the coefficients of $d P P^{-1}$ as $\pi_{i}, \pi_{i j}$ we get as in $\mathbf{1 . 1}$

$$
\omega_{1}=\pi_{1}+d t, \quad \omega_{i}=\pi_{i}+t \pi_{i i} \quad(i=2, \ldots, n) .
$$

Hence a volume element $d V$ of the solid generated by $\mathbf{A}$ is given by

$$
\begin{aligned}
d V & =\left[\omega_{1} \ldots \omega_{n}\right]=\left[\pi_{1}+d t, \pi_{2}+t \pi_{1 n}, \ldots, \pi_{n}+t \pi_{1 n}\right] \\
& =\left[d t \pi_{2} \ldots \pi_{n}\right]+\sum\left[t d t \pi_{12} \pi_{2} \ldots \pi_{n}\right]+\ldots+\left[t^{n-1} d t \pi_{12} \ldots \pi_{1 n}\right] .
\end{aligned}
$$

Integrating this for a domain which is a direct product of a segment $t_{0} \leqq t \leqq$ $t_{0}+a$ and an $n$-dimensional closed orientable domain $D$ with the local parameters $u_{1}, \ldots, u_{n-1}$ in the space of all straight lines as its elements, we get

$$
V=a C_{1}+a^{2} C_{2}+\ldots+a^{n} C_{n}
$$

where we have put

$$
C_{1}=\int\left[\pi_{2} \ldots \pi_{n}\right], C_{2}=1 / 2 \int \sum\left[\pi_{12} \pi_{3} \ldots \pi_{n}\right], \ldots, C_{n}=1 / n \int\left[\pi_{12} \ldots \pi_{1 n}\right],
$$

and $V$ is an algebraic volume of the part swept by the segment $t_{0} \leqq t \leqq t_{0}+a$. Here by an algebraic volume we mean one which is a sum of the volume counted with the sign according to the orientation of the euclidean space and the orientation of the parameter space with point $\left(t, u_{1}, \ldots, u_{n-1}\right)$. Now we assume that for our motion the other end of a unit vector $\mathbf{e}_{1}$ with one end at a fixed point 
covers a domain on a sphere in such a way that its degree of mapping is $k$.

We take $n$ points $P_{i}=\mathbf{A}^{\prime}+a_{i} \mathbf{e}_{1}(i=1, \ldots, n)$ on this line and assume that $\mathbf{A}^{\prime}, P_{i}$ describe $n$-dimensional closed surfaces which enclose solids of volume $V_{0}, V_{i}$ respectively. Now we make one more assumpicn, which I hope to be proved, though diffcult for me. The assumption is that the difference of $V_{0}$ and $V_{i}$ is equal to the algebraic volume of the domain swepi by the segment $A^{\prime} P_{i}$. Then we get by (12)

$$
V_{i}-V_{0}=a_{i} C_{1}+\ldots+a_{i}^{n-1} C_{n-1}+a_{i}^{n} C_{n} .
$$

Eliminating $C_{1}, \ldots, C_{n-1}$ we get

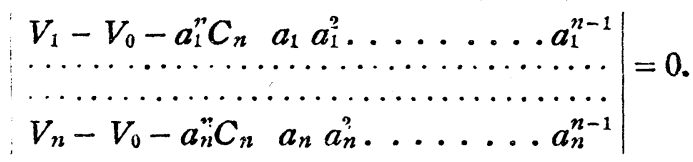

By calculation we get

$$
\sum_{i=0}^{n} V_{i} /\left(d_{0 i} \ldots d_{i-1 i} d_{i+1 i} \ldots d_{n i}\right)=(-1)^{n} C_{n},
$$

where $d_{i j}$ is an oriented distance from $P_{i}$ to $P_{j}$ and $C_{n}=k I_{n}, I_{n}$ being a volume of a unit sphere in the $n$-dimensional space. This is a generalization of Holditch's theorem. If $V_{i}$ 's $(i=1, \ldots, n)$ are all zero, we have $V_{0}=C_{n} d_{10} d_{20} \ldots d_{n 0} . \Lambda$ volume of an ellipsoid is an example of this formula.

2.2 An analogous formula for the spherical case can be obtained, but it takes different forms for $n$ even and for $n$ odd. Moreover since $C_{n}$ is not of the form $k I_{n}$ in this case we eliminate $C_{n}$, too. Thus in the case $n=2$ we get

$$
S_{\mathrm{c}} /\left(d_{01} d_{0:} d_{03}\right)+S_{1} /\left(d_{1} d_{12} d_{13}\right)+S_{:} /\left(d_{\varepsilon c} d_{2}: d_{: 3}\right)+S_{2} /\left(d_{31} d_{31} d_{32}\right)=0
$$

where $S_{i}$ is an area bounded by a curve described by a point $P_{i}$ on a moving great circle, and $d_{j}$ is an oriented length of a chord from $P_{i}$ to $P_{j}$, namely $2 r \sin \left(\alpha_{i j} / 2\right), r$ being a radius of the circle and $\alpha_{i j}$ being an oriented angle corresponding to the arc $P_{i} P_{j}$.

\section{Some formulas about the area}

3.1 Let a triangle $\mathbf{A}_{1} \mathbf{A}_{2} \mathbf{A}_{3}$ move on a euclidean plane and $\mathbf{A}_{0}$ be a point which is relatively fixed to this triangle. Let this motion be represented by a rectangular frame $R=P R_{0}, R_{0}$ being a fundamental frame. Let the coordinates of $A_{i}(i=0,1,2,3)$ with respect to a frame $R$ be $\left(a_{i}, b_{i}\right)$. If we represent the infinitesimal relative displacement of $R$ by $d \mathbf{A}=\pi_{1} \mathbf{e}_{1}+\pi_{2} \mathbf{e}_{2}, d \mathrm{e}_{1}=\pi_{12} \mathbf{e}_{2}, d \mathbf{e}_{2}$ 
$=-\pi_{12} \mathbf{e}_{1}$, then the coefficients of the relative displacement of $R_{i}$ with $\mathbf{A}_{i}$ as its origin and $\mathbf{e}_{1}, \mathbf{e}_{2}$ as its vectors are given by

$$
\omega_{1}=\pi_{1}-b_{i} \pi_{12}, \quad \omega_{2}=\pi_{2}+a_{i} \pi_{12}, \quad \omega_{12}=\pi_{12} .
$$

If the motion of $\mathbf{A}_{0} \mathbf{A}_{1} \mathbf{A}_{2} \mathbf{A}_{3}$ is two-parametric, the areal element of the domains swept by $\mathbf{A}_{i}$ is given by

$$
d S=\left[\omega_{1} \omega_{2}\right]=\left[\pi_{1} \pi_{2}\right]+a_{i}\left[\pi_{1} \pi_{12}\right]+b_{i}\left[\pi_{2} \pi_{12}\right] .
$$

We denote by $S_{i}$ an algebraic area swept by $A_{i}$. Then we get

$$
S_{i}=\int\left[\pi_{1} \pi_{2}\right]+a_{i} \int\left[\pi_{1} \pi_{12}\right]+b_{i} \int\left[\pi_{2} \pi_{12}\right] \text {. }
$$

Hence eliminating $\int\left[\pi_{1} \pi_{2}\right], \int\left[\pi_{1} \pi_{12}\right], \int\left[\pi_{2} \pi_{12}\right]$ we get

$$
\left|\begin{array}{llll}
S_{0} & 1 & a_{0} & b_{0} \\
S_{1} & 1 & a_{1} & b_{1} \\
S_{2} & 1 & a_{2} & b_{2} \\
S_{3} & 1 & a_{3} & b_{3}
\end{array}\right|=0 .
$$

This can be written in the form

$$
\sum_{i=0}^{3} S_{i} \sigma_{i}=0
$$

where $\sigma_{i}$ is an oriented area of the triangle with the vertices except $A_{i}$.

3. 2 We take a curve on a euclidean plane and denote by $s$ an arc lengih from a fixed point on it to an arbitrary point on it and by $k$ a curvature. From each point of the curve we draw a segment of a length $l$ and let $\theta$ be an angle which the segmen $\imath$ makes with the tangent of the curve at the point. Then for an algebraic area swept by the segmen: we get by calculation

$$
d S=\int l \sin \theta d s-\frac{1}{2} \int l^{2}(k d s+d \theta) .
$$

We can apply this to the area $S$ bounded by a curve $C$ and a roulette. Here we mean by a roulette a curve described by a point on a closed curve $C_{0}$ which rolls on $C$ without slipping and we take into consideration an arc of the curve corresponding to one circulation of $C_{0}$, two ends being on $C$. Let $S_{\mathrm{J}}$ be an area bounded by $C_{0}$ and $k, k_{0}$ be curvatures of $C, C_{0}$ at the point of contact which we represent as functions of the common arc length $s$, while $l$ is a distance from a point on the roulette to a corresponding point of contact. Then by calculation we get from (16) under a certain assumption

$$
S=S_{0}+\frac{1}{2} \int l^{2}\left(k_{0}-k\right) d s .
$$


Here we assume that the area $S_{0}$ of the domain bounded by $C_{0}$ is swept positively by a chord drawn from the point on $C_{0}$ at which $C_{0}$ touches $C$ at the initial state to a point on $C_{0}$ of contact with $C$ at each instant. Especially if $C_{0}$ is a circle of radius $r$, we get

$$
S=3 \pi r^{2}-\alpha r^{2}+\int r^{2} \cos 2 \theta \cdot k d s,
$$

where the curvature $1 / \boldsymbol{r}$ of the circle $C_{0}$ is counted positively and the curvature $k$ of $C$ is of the sign corresponding to that of $C_{0}$, while $\alpha=\int k d s$ is an angle between the two tangents of $C$ at the both ends.

About the area on the unit sphere we can obtain a formula analogous to (16). We take notations as in (16) and let $\theta$ be an angle in which the center of the sphere commands an arc of a great circle drawn from each point on the curve. Then we get

$$
S=\int \sin \alpha \sin \theta d s-\int(1-\operatorname{con} \alpha)(k d s+d \theta)
$$

\section{REFERENCES}

[1] H. Weyl, On the volume of tubes, Amer. J. Math. 61 (1939).

[2] E. Cartan, Leçons sur la géométrie des espaces de Riemann, Paris, 1946.

Mathematical Institute, Nagoya University 